\title{
Selective synthesis of functionalized allyl amines and ethers from (E)-2,3-bisphosphonated allyl bromide
}

\author{
Maha Ameur and Hassen Amri * \\ Selective Organic Synthesis \& Biological Activity, Faculty of Science, \\ University of Tunis El Manar, 2092 Tunis, Tunisia
}

\begin{abstract}
An efficient and straightforward approach toward functionalized allylic amines and ethers is disclosed starting from tetraethyl (3-bromoprop-1-ene-1,2-diyl)bisphosphonate (E)-1 and secondary and tertiary amines. The coupling reaction of allyl bromide $(\boldsymbol{E})$-1 with less bulky secondary amines provides a new family of allylamines $\mathbf{3}$, while, its reaction with more bulky secondary and tertiary amines, in methanol at reflux, led to functional allyl ethers $\mathbf{4}$ and $\mathbf{5}$.
\end{abstract}

Keywords: allyl bromide; secondary amine; tertiary amine; allylamine; allyl ether.

\section{Introduction}

Heteroatom-containing compounds are important synthetic targets of interest in organic and medicinal chemistry ${ }^{1-8}$. This is mainly because they are useful intermediates for the synthesis of biologically naturally occurring products ?, antibiotics ${ }^{10}$, chiral auxiliaries ${ }^{11}$ and $\beta$-amino alcohols ${ }^{12}$. Despite, the different methodologies discovered for the synthesis of carbon- heteroatom bonds formation reactions, much of them have many constraints such as harsh reaction conditions, high cost of reagents and long reaction times. Among these various potentialities, the addition of an amine to an unsaturated $\mathrm{C}-\mathrm{C}$ bond provides an excellent route for the formation of carbon-heteroatom bond. Following this protocol, our research group has been actively working on the synthesis of new compounds containing $\mathrm{C}-\mathrm{N}$ and $\mathrm{C}-\mathrm{O}$ bonds ${ }^{13}$. In pursuit of our studies, the present paper reports an efficient protocol for the synthesis of novel functionalized allylamines 3, allyl ethers $\mathbf{4}$ and $\mathbf{5}$ through the reaction of tetraethyl (prop-2-ene-1,2-diyl)bisphosphonate $(\boldsymbol{E})-\mathbf{1}$ with secondary and tertiary amines (Figure 1)<smiles>CCOC(=O)P=C(CBr)PCCO</smiles>
( $E)-1$<smiles>[R12]NC(C(=C)[P+](=O)OCC)[P+](=O)OCC</smiles><smiles>C=[PH](OCC)C(=CC(=O)OCC)COC</smiles><smiles>C=C(OCC)C(OC)P(=O)(OCC)OCC</smiles>

Figure 1. Structure of the new functionalized allylamines $\mathbf{3}$, allyl ethers $\mathbf{4}$ and $\mathbf{5}$

\section{Results and Discussion}

In our recent work, we have developed a simple and convenient synthesis of tetraethyl (3-bromoprop1-ene-1,2-diyl)bisphosphonate $(\boldsymbol{E})-\mathbf{1}^{14}$ via a tandem reaction of bromination-dehydrobromination of tetraethyl (prop-2-ene-1,2-diyl)bisphosphonate in the presence of $\mathrm{DBU}$ in acetonitrile at room temperature (Scheme 1). 


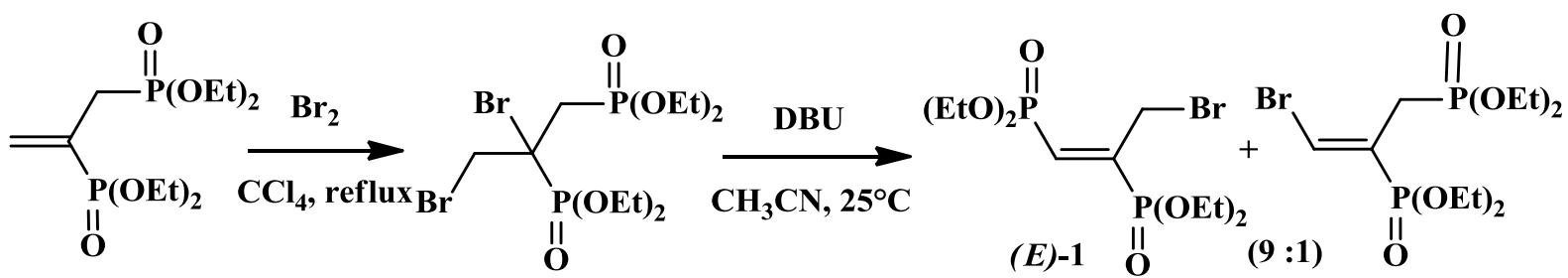

Scheme 1. Synthesis of the mixture of allyl and vinyl bromides

In a recent work ${ }^{14}$ devoted to the study of the reactivity of allyl bromide $(\boldsymbol{E})-\mathbf{1}$ with various primary amines in methanol at $0{ }^{\circ} \mathrm{C}$, we have shown that the coupling reaction follows an additionelimination or $\mathrm{S}_{\mathrm{N}} 2$, process to provide a new family of functionalized allyl amines $\mathbf{3}$ with good yields (Scheme 2). Allylamines and their derivatives have proven to be valuable synthons in organic chemistry ${ }^{15}$ as they are found in a range of natural products such as gabaculine ${ }^{16}$, ocyzosymicine ${ }^{17}$ and cytosinine ${ }^{18}$ and serve as precursors to a large number of pharmaceutically and biologically active compounds which display a variety of pharmacological properties including anti-convulsant ${ }^{19}$, antiinflammatory ${ }^{20}$ and anti-tumor agents ${ }^{21}$.
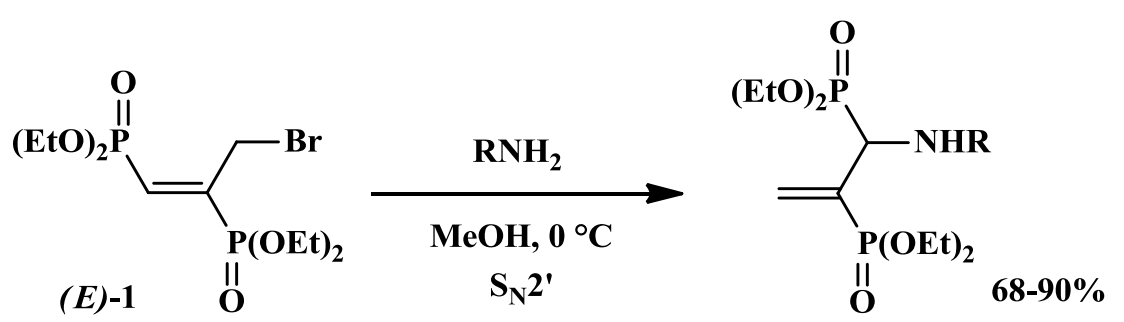

Scheme 2. Synthesis of tetraethyl (1-(alkylamino)prop-2-ene-1,2-diyl)bisphosphonates

This interesting result prompted us to study the behavior of allyl bromide $(\boldsymbol{E})$-1 towards a series of secondary and tertiary amines. We started our investigation using excess amounts of less bulky secondary amines ( 2 equiv.) in methanol at room temperature. Thus, their coupling reaction with allyl bromide $(\boldsymbol{E})$-1 afforded a new family of allylamines 3a-c via an expected $\mathrm{S}_{\mathrm{N}} 2$ ' substitution in good yields ranging from 82 to $87 \%$ (Scheme 3, Table 1).<smiles>CCOP(=O)(C=C(CBr)PCCOC)OCC</smiles>

(E)-1 $\stackrel{\text { II }}{\mathrm{O}}$

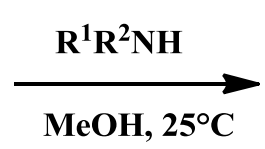

$\mathrm{MeOH}, 25^{\circ} \mathrm{C}$

Scheme 3. Preparation of functionalized allylamines 3a-c

Table 1. Synthesis of tetraethyl (1-(dialkylamino)prop-2-ene-1,2-diyl)bisphosphonates 3a-c.

\begin{tabular}{cccccc}
\hline Entry & $\mathbf{R}^{1}$ & $\mathbf{R}^{2}$ & Product & Time (h) & Yield (\%) \\
\hline 1 & $\mathrm{Me}$ & $\mathrm{Me}$ & $\mathbf{3 a}$ & 3 & 87 \\
2 & $\mathrm{Et}$ & $\mathrm{Et}$ & $\mathbf{3 b}$ & 5 & 85 \\
3 & ${ }^{n} \mathrm{Pr}$ & ${ }^{n} \mathrm{Pr}$ & $\mathbf{3 c}$ & 7 & 82 \\
\hline
\end{tabular}

\footnotetext{
${ }^{*}$ Yields refer to the pure isolated products characterized by ${ }^{1} \mathrm{H},{ }^{13} \mathrm{C}$ NMR.
}

In contrast, with more bulky secondary amines such as diisopropylamine, dicyclohexylamine and diphenylamine in refluxing methanol, the reaction led to unexpected methoxy allylether $(\boldsymbol{E})-\mathbf{4}$, resulting from an unpredictable successive $\mathrm{S}_{\mathrm{N}} 2^{\prime}-\mathrm{S}_{\mathrm{N}} 2$ ' reactions (Scheme 4). The results are gathered in Table 2. 


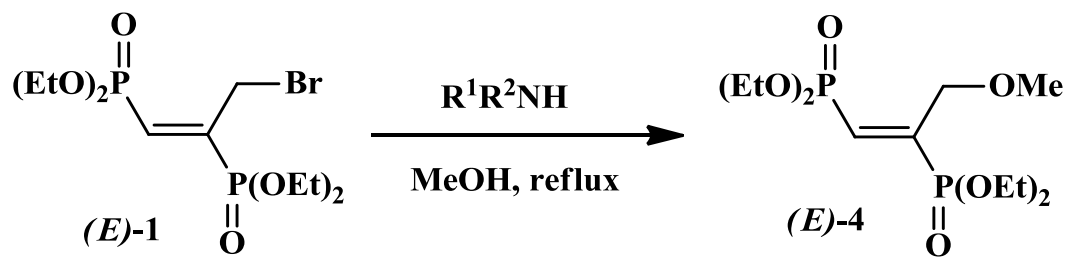

Scheme 4. Unpredictable synthesis of allyl ether $(\boldsymbol{E}) \mathbf{- 4}$.

Table 2. Synthesis of functionalized primary methoxy allyl ether $(\boldsymbol{E}) \mathbf{- 4}$.

\begin{tabular}{cccccc}
\hline Entry & $\mathbf{R}^{1}$ & $\mathbf{R}^{2}$ & Product & Time (h) & Yield (\%) $^{*}$ \\
\hline 1 & ${ }^{i} \mathrm{Pr}$ & ${ }^{c} \mathrm{C}_{6} \mathrm{H}_{11}$ & 4 & 5 & 72 \\
2 & ${ }^{c} \mathrm{C}_{6} \mathrm{H}_{11}$ & ${ }^{c} \mathrm{C}_{6} \mathrm{H}_{11}$ & 4 & 8 & 74 \\
3 & $\mathrm{Ph}$ & $\mathrm{Ph}$ & 4 & 15 & 78 \\
\hline
\end{tabular}

${ }^{*}$ Yields refer to the pure isolated products characterized by ${ }^{1} \mathrm{H},{ }^{13} \mathrm{C}$ NMR

Mechanistically, the formation of allyl ether $(\boldsymbol{E})$ 4 can be achieved from the brominated derivative $(\boldsymbol{E})$-1 through successive $\mathrm{S}_{\mathrm{N}} 2$ '-type reaction with bulky secondary amines. First, secondary amine played the role of co-nucleophile in a direct nucleophilic substitution of allyl bromide $(\boldsymbol{E})-\mathbf{1}$ to give the corresponding quaternary ammonium salt. Then, this intermediate undergoes a second $\mathrm{S}_{\mathrm{N}} 2$ ' reaction with methanol which plays a dual role as reactant and as solvent (Scheme 5).

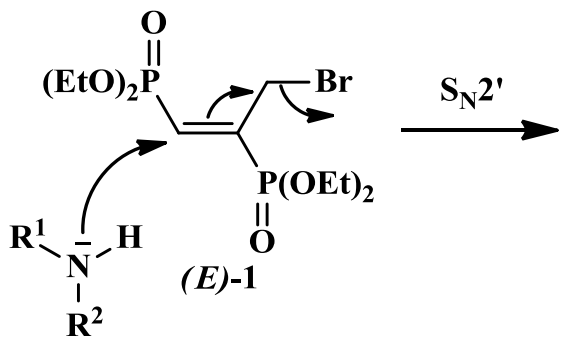

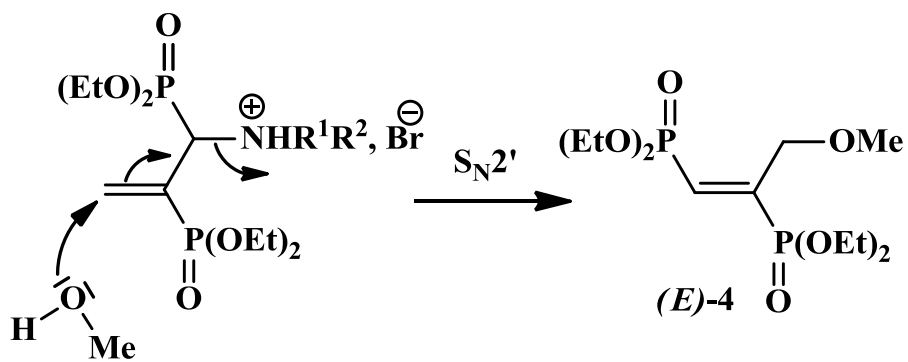

Scheme 5. Plausible mechanism for the formation of allyl ether $(\boldsymbol{E})-\mathbf{4}$

The structure of $\mathbf{4}$ was established on the basis of its ${ }^{1} \mathrm{H}$ and ${ }^{13} \mathrm{C}$ NMR spectra and by heteronuclear multiple bond correlation (HMBC). The (E)stereochemistry was assigned on the basis of NOESY spectra that show no correlation between the ethylenic proton $(6.71 \mathrm{ppm})$ and those of group $\mathrm{CH}_{2} \mathrm{OMe}(\delta=4.49 \mathrm{ppm})$. This result indicates that the vinylic proton and the $\mathrm{CH}_{2}$ group are on opposite sides of the double bond and therefore, the alkene in 4 is $(E)$ configuration. The obtained result may be explained by the steric factor of secondary amines. The results of the electrophilic behavior of allyl bromide $(\boldsymbol{E})-1$ towards different more bulky and less bulky secondary amines are summarized in Scheme 6.

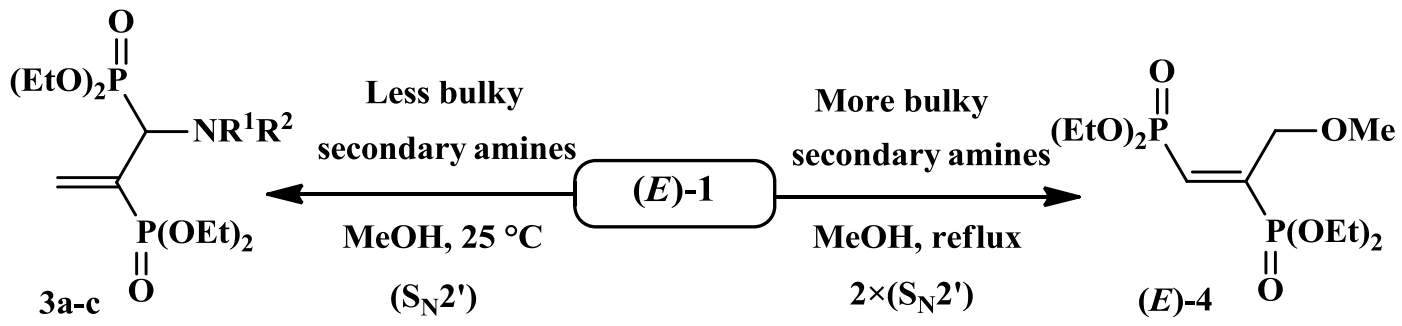

Scheme 6. Regioselective substitution of allyl bromide $(\boldsymbol{E})-\mathbf{1}$ with some secondary amines

The diversity of previous results obtained with less or more bulky secondary amines prompted us to try other more hindered nitrogen nucleophiles such as tertiary amines (triethylamine, ethyl diisopropyl- 
amine and triethanolamine). Surprisingly, it appears that the size of these amines strongly influences their reaction course with allyl bromide $(\boldsymbol{E})-\mathbf{1}$, while the coupling is only possible in refluxing methanol to yield a representative of secondary methoxy allyl ether: tetraethyl (1-methoxyprop-2-ene-1,2-diyl) bisphosphonate $\mathbf{5}$ in moderate to good yields (Scheme 7, Table 3).
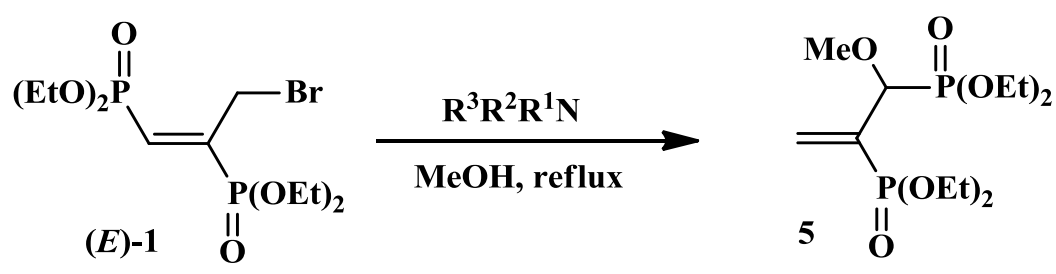

Scheme 7. Synthesis of tetraethyl (1-methoxyprop-2-ene-1,2-diyl)bisphosphonate 5

Table 3: One pot synthesis of secondary allyl ether $\mathbf{5}$.

\begin{tabular}{|c|c|c|c|c|c|c|}
\hline Entry & $\mathbf{R}^{1}$ & $\mathbf{R}^{2}$ & $\mathbf{R}^{3}$ & Product & Time (d) & Yield $(\%)$ \\
\hline 1 & $\mathrm{Et}$ & $\mathrm{Et}$ & $\mathrm{Et}$ & 5 & 3 & 84 \\
\hline 2 & $\mathrm{Et}$ & ${ }^{i} \operatorname{Pr}$ & ${ }^{i} \operatorname{Pr}$ & 5 & 6 & 78 \\
\hline 3 & $\left(\mathrm{CH}_{2}\right)_{2} \mathrm{OH}$ & $\left(\mathrm{CH}_{2}\right)_{2} \mathrm{OH}$ & $\left(\mathrm{CH}_{2}\right)_{2} \mathrm{OH}$ & 5 & 5 & 68 \\
\hline
\end{tabular}

\footnotetext{
${ }^{*}$ Yields refer to the pure isolated products characterized by ${ }^{1} \mathrm{H},{ }^{13} \mathrm{C}$ NMR
}

The plausible mechanism which illustrates the synthesis of secondary methoxy allyl ether $\mathbf{5}$ is depicted in Scheme 8. The reaction consisted of a two-step sequence: substitution $\mathrm{S}_{\mathrm{N}} 2$-type reaction of $(\boldsymbol{E})$-1 with tertiary amines followed an easy displacement of the resulting quaternary ammonium by methanol in a clean $\mathrm{S}_{\mathrm{N}} 2$ ' reaction to provide the expected product 5. Taking into account of the limited methanol nucleophilicity, the substitution of allyl bromide $(\boldsymbol{E}) \mathbf{- 1}$ with this solvent is very slow and inefficient even at reflux. The use of more hindered secondary and tertiary amines as conucleophilic nitrogen reagents is to provide a quaternary ammonium which can be easily displaced by the methanol.

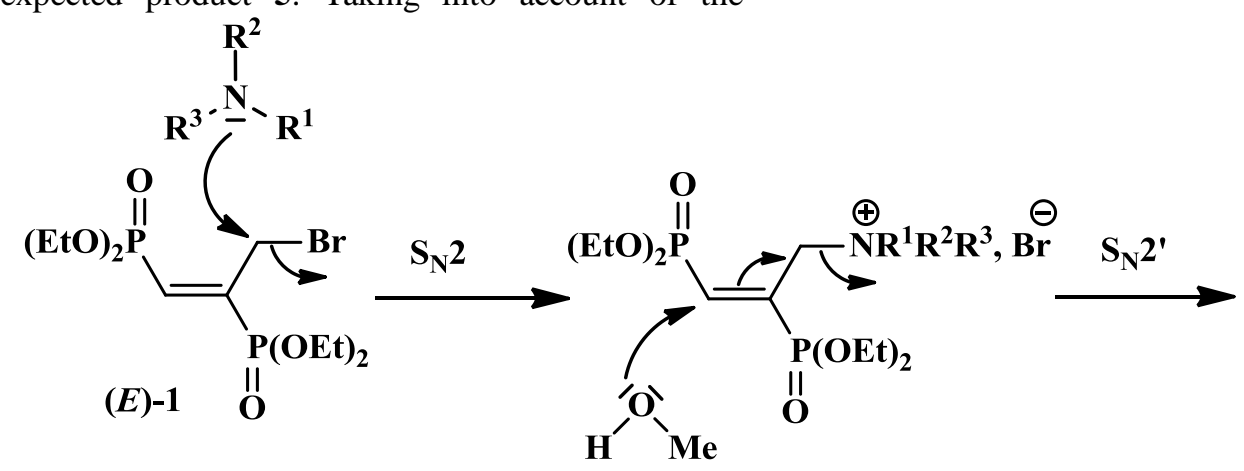<smiles>C=C(C(=O)OCC)[P+](=O)OCC</smiles>

Scheme 8. Plausible mechanism for the synthesis of allyl ether $\mathbf{5}$

\section{Conclusion}

In conclusion, we have successfully developed an efficient and highly regio- and stereoselective synthesis of functionalized allyl amines $\mathbf{3}$ and allyl ethers $\mathbf{4}$ and $\mathbf{5}$ via an effective coupling reaction of allyl bromide $(\boldsymbol{E})-1$ with a variety of secondary and tertiary amines under mild conditions.

\section{Experimental Section}

Starting materials and solvents were used without further purification. ${ }^{1} \mathrm{H}-\mathrm{NMR},{ }^{31} \mathrm{P}-\mathrm{NMR}$ and ${ }^{13} \mathrm{C}$-NMR spectra were recorded on a Bruker AMX 300 spectrometer working at $300 \mathrm{MHz}, 121 \mathrm{MHz}$ and $75 \mathrm{MHz}$ respectively for ${ }^{1} \mathrm{H},{ }^{31} \mathrm{P}$ and ${ }^{13} \mathrm{C}$ with $\mathrm{CDCl}_{3}$ as the solvent and TMS as the internal standard. 
The chemical shifts $(\delta)$ and coupling constants $(J)$ are, respectively, expressed in parts per million (ppm) and Hertz (Hz). All NMR spectra were acquired at room temperature. Assignments of proton $\left({ }^{1} \mathrm{H}-\mathrm{NMR}\right)$ and carbon $\left({ }^{13} \mathrm{C}-\mathrm{NMR}\right)$ signals were secured by DEPT 135 and HMBC experiments. Multiplicity of peaks is indicated by the following: s, singlet; d, doublet; t, triplet; dd, double doublet and $\mathrm{m}$, multiplet. IR spectra were recorded on a Bruker Vertex 70 FT-IR spectrophotometer. HighResolution Mass Spectrometry (HRMS) analyses were performed in Laberca laboratory at Oniris (Nantes-Atlantic National College of Veterinary Medicine, Food Science and Engineering) on a mass spectrometer equipped with a door coupled to a linear Orbitrap (LTQ-Orbitrap of Thermo Fisher Scientific) in positive electrospray ionization. All reactions were monitored by TLC on silica gel plates (Fluka Kieselgel $60 \mathrm{~F}_{254}$, Merck) eluting with the solvents indicated, visualized by a $254 \mathrm{~nm}$ UV lamp and aqueous potassium permanganate solution. For column chromatography, Fluka Kieselgel 70-230 mesh was used.

General Procedure for the synthesis of tetraethyl1-(dialkylamino)prop-2-ene-1,2-diyl)bisphosphonates 3a-c

To a solution of tetraethyl (3-bromoprop-1-ene1,2-diyl)bisphosphonate $\quad \boldsymbol{E}-\mathbf{1} \quad(0.2 \mathrm{~g}, \quad 0.5 \quad \mathrm{mmol})$ diluted in $5 \mathrm{~mL}$ of absolute methanol was added dropwise secondary amine $(2 \mathrm{mmol})$ diluted in $3 \mathrm{~mL}$ of methanol at $0{ }^{\circ} \mathrm{C}$. The reaction mixture was stirred at room temperature. After stirring during the time indicated in Table 1, the excess of the solvent was evaporated under reduced pressure and the crude product was purified by chromatography on silica gel $\left(\mathrm{CH}_{2} \mathrm{Cl}_{2}-\mathrm{MeOH}, 9.5-0.5\right)$.

\section{Tetraethyl(1-(dimethylamino)prop-2-ene-1,2- diyl)bisphosphonate 3a \\ Yield: $87 \%$; Yellow liquid;}

IR (neat): $1650(\mathrm{C}=\mathrm{C}), 1243(\mathrm{P}=\mathrm{O})$.

${ }^{1} \mathbf{H}$-NMR $\left(\mathbf{C D C l}_{3}, \boldsymbol{\delta}\right): 6.56\left(\mathrm{~d}, 1 \mathrm{H},{ }^{3} J_{H P}=47.7 \mathrm{~Hz}\right.$, $=\mathrm{CH}) ; 6.44\left(\mathrm{dd}, 1 \mathrm{H},{ }^{3} J_{H P}=23 \mathrm{~Hz},{ }^{4} J_{H P}=3 \mathrm{~Hz}\right.$, $=\mathrm{CH}) ; 4.25-4.01\left(\mathrm{~m}, 8 \mathrm{H}, 4 \mathrm{OCH}_{2}\right) ; 3.88\left(\mathrm{dd}, 1 \mathrm{H},{ }^{2} J_{H P}\right.$ $\left.=24 \mathrm{~Hz},{ }^{3} J_{H P}=15 \mathrm{~Hz}, \mathrm{CHP}\right) ; 2.47\left(\mathrm{~s}, 3 \mathrm{H}, \mathrm{CH}_{3}\right)$; $2.46\left(\mathrm{~s}, 3 \mathrm{H}, \mathrm{CH}_{3}\right) ; 1.33,1.28(2 \mathrm{t}, 12 \mathrm{H}, J=6 \mathrm{~Hz}$, $\left.J=6 \mathrm{~Hz}, 4 \mathrm{CH}_{3}\right)$;

${ }^{13} \mathbf{C}-N M R\left(\mathbf{C D C l}_{3}, \boldsymbol{\delta}\right): 135.8\left(\mathrm{t},=\mathrm{CH}_{2},{ }^{2} J_{C P}={ }^{3} J_{C P}=\right.$ $7.5 \mathrm{~Hz}) ; 131.4\left(\mathrm{dd},=\mathrm{C},{ }^{1} J_{C P}=172.5 \mathrm{~Hz},{ }^{2} J_{C P}=11.5\right.$ $\mathrm{Hz}$ ); 63.1, $62.7\left(2 \mathrm{~d}, 2 \mathrm{OCH}_{2},{ }^{2} J_{C P}=7.5 \mathrm{~Hz},{ }^{2} J_{C P}=7.5\right.$ $\mathrm{Hz}) ; 62.3,62.0\left(2 \mathrm{~d}, 2 \mathrm{OCH}_{2},{ }^{2} J_{C P}=6 \mathrm{~Hz},{ }^{2} J_{C P}=6\right.$ $\mathrm{Hz}) ; 60.2\left(\mathrm{dd}, \mathrm{CHP},{ }^{1} J_{C P}=156.75 \mathrm{~Hz},{ }^{2} J_{C P}=11.25\right.$ $\mathrm{Hz}) ; 42.9\left(\mathrm{~s}, \mathrm{CH}_{3}\right) ; 42.8\left(\mathrm{~s}, \mathrm{CH}_{3}\right) ; 16.5,16.4(2 \mathrm{~d}$, $\left.2 \mathrm{CH}_{3},{ }^{3} J_{C P}=6.75 \mathrm{~Hz},{ }^{3} J_{C P}=6.75 \mathrm{~Hz}\right) ; 16.3,16.2$ $\left(2 \mathrm{~d}, 2 \mathrm{CH}_{3},{ }^{3} J_{C P}=6 \mathrm{~Hz},{ }^{3} J_{C P}=6 \mathrm{~Hz}\right)$;

${ }^{31} \mathbf{P}$-NMR $\left(\mathbf{C D C l}_{3}, \boldsymbol{\delta}\right): 17.9(\mathrm{~d},=\mathrm{CP}, J=58.08 \mathrm{~Hz})$; 21.9 (d, CHP, $J=58.08 \mathrm{~Hz}$ );

HRMS (ESI+) calculated for $\left[\mathrm{M}+\mathrm{H}^{+}\right] 358.15429$, found 358.15359 .
Tetraethyl(1-(diethylamino)prop-2-ene-1,2diyl)bis-phosphonate $\mathbf{3 b}$

Yield: 85\%; Yellow liquid;

IR (neat): $1648(\mathrm{C}=\mathrm{C}), 1233(\mathrm{P}=\mathrm{O})$.

${ }^{1} \mathbf{H}-\mathbf{N M R}\left(\mathbf{C D C l}_{3}, \boldsymbol{\delta}\right): 6.58\left(\mathrm{~d}, 1 \mathrm{H},{ }^{3} J_{H P}=48 \mathrm{~Hz}\right.$, $=\mathrm{CH}) ; 6.35\left(\mathrm{dd}, 1 \mathrm{H},{ }^{3} J_{H P}=24 \mathrm{~Hz},{ }^{4} J_{H P}=3 \mathrm{~Hz}\right.$, $=\mathrm{CH}) ; 4.20-3.92\left(\mathrm{~m}, 9 \mathrm{H}, \mathrm{CHP}+4 \mathrm{OCH}_{2}\right) ; 2.97-2.74$ $\left(\mathrm{m}, 2 \mathrm{H}, \mathrm{CH}_{2}\right) ; 2.58-2.47\left(\mathrm{~m}, 2 \mathrm{H}, \mathrm{CH}_{2}\right) ; 1.27(3 \mathrm{t}, 9 \mathrm{H}$, $\left.J=6 \mathrm{~Hz}, 3 \mathrm{CH}_{3}\right) ; 1.19\left(\mathrm{t}, 3 \mathrm{H}, J=6 \mathrm{~Hz}, \mathrm{CH}_{3}\right) ; 1.00$ (t, $\left.6 \mathrm{H}, J=6 \mathrm{~Hz}, 2 \mathrm{CH}_{3}\right)$;

${ }^{13} \mathbf{C}$-NMR $\left(\mathbf{C D C l}_{\mathbf{3}}, \boldsymbol{\delta}\right): 136.4\left(\mathrm{t},=\mathrm{CH}_{2},{ }^{2} \boldsymbol{J}_{C P}={ }^{3} J_{C P}=\right.$ $6.75 \mathrm{~Hz}) ; 132.4\left(\mathrm{dd},=\mathrm{C},{ }^{1} J_{C P}=170.25 \mathrm{~Hz},{ }^{2} J_{C P}=\right.$ $10.5 \mathrm{~Hz},) ; 63.1,62.8\left(2 \mathrm{~d}, 2 \mathrm{OCH}_{2},{ }^{2} J_{C P}=7.5 \mathrm{~Hz},{ }^{2} J_{C P}\right.$ $=7.5 \mathrm{~Hz}) ; 62.4,62.0\left(2 \mathrm{~d}, 2 \mathrm{OCH}_{2},{ }^{2} J_{C P}=7.5 \mathrm{~Hz},{ }^{2} J_{C P}\right.$ $=7.5 \mathrm{~Hz}) ; 55.5\left(\mathrm{dd}, \mathrm{CHP},{ }^{1} J_{C P}=159 \mathrm{~Hz},{ }^{2} J_{C P}=\right.$ $11.25 \mathrm{~Hz}) ; 45.1\left(\mathrm{~s}, \mathrm{CH}_{2}\right) ; 45.1\left(\mathrm{~s}, \mathrm{CH}_{2}\right) ; 16.6,16.4$ $\left(2 \mathrm{~d}, 2 \mathrm{CH}_{3},{ }^{3} J_{C P}=6.5 \mathrm{~Hz},{ }^{3} J_{C P}=6.5 \mathrm{~Hz}\right) ; 16.3,16.2$ $\left(2 \mathrm{~d}, 2 \mathrm{CH}_{3},{ }^{3} J_{C P}=6 \mathrm{~Hz},{ }^{3} J_{C P}=6 \mathrm{~Hz}\right) ; 14.0\left(\mathrm{~s}, \mathrm{CH}_{3}\right)$; $14.0\left(\mathrm{~s}, \mathrm{CH}_{3}\right)$;

${ }^{31} \mathbf{P}-\mathbf{N M R}\left(\mathbf{C D C l}_{3}, \boldsymbol{\delta}\right): 18.5(\mathrm{~d},=\mathrm{CP}, J=62.92 \mathrm{~Hz})$; 22.6 (d, CHP, $J=62.92 \mathrm{~Hz}$ );

HRMS (ES+) calculated for $\left[\mathrm{M}+\mathrm{Na}^{+}\right]$408.1681, found 408.1684 .

\section{(1-(dipropylamino)prop-2-ene-1,2-diyl)bis- phosphonate 3c}

Yield: $82 \%$; Yellow liquid;

IR (neat): $1642(\mathrm{C}=\mathrm{C}), 1244(\mathrm{P}=\mathrm{O})$;

${ }^{1} \mathbf{H}-\mathbf{N M R}\left(\mathbf{C D C l}_{3}, \boldsymbol{\delta}\right): 6.60\left(\mathrm{~d}, 1 \mathrm{H},{ }^{3} J_{H P}=48 \mathrm{~Hz}\right.$, $=\mathrm{CH}) ; 6.50\left(\mathrm{dd}, 1 \mathrm{H},{ }^{3} J_{H P}=21 \mathrm{~Hz},{ }^{4} J_{H P}=3 \mathrm{~Hz}\right.$, $=\mathrm{CH}) ; 4.22-3.94\left(\mathrm{~m}, 9 \mathrm{H}, \mathrm{CHP}+4 \mathrm{OCH}_{2}\right) ; 2.76(\mathrm{t}$, $\left.4 \mathrm{H}, J=6 \mathrm{~Hz}, 2 \mathrm{CH}_{2}\right) ; 1.67-1.56\left(\mathrm{~m}, 4 \mathrm{H}, 2 \mathrm{CH}_{2}\right)$; $1.30,1.22\left(2 \mathrm{t}, 12 \mathrm{H}, J=6.5 \mathrm{~Hz}, J=6.5 \mathrm{~Hz}, 4 \mathrm{CH}_{3}\right)$; $0.90\left(\mathrm{t}, 6 \mathrm{H}, J=6 \mathrm{~Hz}, 2 \mathrm{CH}_{3}\right)$;

${ }^{13} \mathbf{C}-\mathrm{NMR}\left(\mathbf{C D C l}_{\mathbf{3}}, \boldsymbol{\delta}\right): 137.4\left(\mathrm{t},=\mathrm{CH}_{2},{ }^{2} J_{C P}={ }^{3} J_{C P}=\right.$ $7.5 \mathrm{~Hz}) ; 133.5\left(\mathrm{dd},=\mathrm{C},{ }^{1} J_{C P}=171.5 \mathrm{~Hz},{ }^{2} J_{C P}=11.5\right.$ $\mathrm{Hz},) ; 63.4,62.9\left(2 \mathrm{~d}, 2 \mathrm{OCH}_{2},{ }^{2} J_{C P}=7.5 \mathrm{~Hz},{ }^{2} J_{C P}=7.5\right.$ $\mathrm{Hz}) ; 62.5,62.1\left(2 \mathrm{~d}, 2 \mathrm{OCH}_{2},{ }^{2} J_{C P}=7.5 \mathrm{~Hz},{ }^{2} J_{C P}=7.5\right.$ $\mathrm{Hz}) ; 55.4\left(\mathrm{dd}, \mathrm{CHP},{ }^{l} J_{C P}=161 \mathrm{~Hz},{ }^{2} J_{C P}=11.5 \mathrm{~Hz}\right)$; $45.2\left(\mathrm{~s}, \mathrm{CH}_{2}\right) ; 45.1\left(\mathrm{~s}, \mathrm{CH}_{2}\right) ; 16.6,16.5\left(2 \mathrm{~d}, 2 \mathrm{CH}_{3}\right.$, $\left.{ }^{3} J_{C P}=7.5 \mathrm{~Hz},{ }^{3} J_{C P}=7.5 \mathrm{~Hz}\right) ; 16.3,16.3\left(2 \mathrm{~d}, 2 \mathrm{CH}_{3}\right.$, $\left.{ }^{3} J_{C P}=6 \mathrm{~Hz},{ }^{3} J_{C P}=6 \mathrm{~Hz}\right) ; 11.0\left(\mathrm{~s}, \mathrm{CH}_{3}\right) ; 11.0$ (s, $\left.\mathrm{CH}_{3}\right)$;

${ }^{31} \mathbf{P}-\mathbf{N M R}\left(\mathbf{C D C l}_{3}, \boldsymbol{\delta}\right): 18.7(\mathrm{~d},=\mathrm{CP}, J=61.42 \mathrm{~Hz})$; 22.9 (d, CHP, $J=61.42 \mathrm{~Hz}$ );

HRMS (ES+) calculated for $\left[\mathrm{M}+\mathrm{Na}^{+}\right]$436.1994, found 436.1997 .

General Procedure for the synthesis of tetraethyl(3-methoxyprop-1-ene-1,2-diyl)bisphosphonate 4

Compound 4 was similarly prepared as 3a-c from $(\boldsymbol{E})$-1. The reaction mixture was stirred in refluxed methanol for 5 to 15 hours (Table 2). The crude product 4 was purified by chromatography on silica gel $\left(\mathrm{CH}_{2} \mathrm{Cl}_{2}-\mathrm{MeOH}, 9.4-0.6\right)$.

Yellow liquid;

${ }^{1} \mathbf{H}-\mathbf{N M R}\left(\mathbf{C D C l}_{\mathbf{3}}, \boldsymbol{\delta}\right): 6.71\left(\mathrm{dd}, 1 \mathrm{H},{ }^{2} J_{H P}=27 \mathrm{~Hz}\right.$, $\left.{ }^{3} J_{H P}=15 \mathrm{~Hz},=\mathrm{CH}\right) ; 4.49\left(\mathrm{~d}, 2 \mathrm{H},{ }^{3} J_{H P}=15 \mathrm{~Hz}\right.$, $\left.\mathrm{CH}_{2} \mathrm{OCH}_{3}\right)$; 4.20- 4.08 (m, 8H, 4OCH $) ; 3.39$ (s, $\left.\mathrm{OCH}_{3}\right) ; 1.34,1.28(2 \mathrm{t}, 12 \mathrm{H}, J=6 \mathrm{~Hz}, J=6 \mathrm{~Hz}$, $\left.4 \mathrm{CH}_{3}\right)$; 
${ }^{13} \mathbf{C}$-NMR $\left(\mathbf{C D C l}_{\mathbf{3}}, \boldsymbol{\delta}\right): 148.2\left(\mathrm{dd},=\mathrm{C},{ }^{1} J_{C P}=164.25\right.$ $\left.\mathrm{Hz},{ }^{2} J_{C P}=7.5 \mathrm{~Hz}\right) ; 133.1\left(\mathrm{dd},=\mathrm{CH},{ }^{1} J_{C P}=172.5 \mathrm{~Hz}\right.$, $\left.{ }^{2} J_{C P}=9.75 \mathrm{~Hz}\right) ; 68.0\left(\mathrm{t}, \mathrm{CH}_{2} \mathrm{OCH}_{3},{ }^{2} J_{C P}={ }^{3} J_{C P}=9\right.$ $\mathrm{Hz}) ; 62.7\left(\mathrm{~d}, 2 \mathrm{OCH}_{2},{ }^{2} J_{\mathrm{CP}}=6 \mathrm{~Hz}\right) ; 62.4\left(\mathrm{~d}, 2 \mathrm{OCH}_{2}\right.$, $\left.{ }^{2} J_{C P}=6 \mathrm{~Hz}\right) ; 58.6\left(\mathrm{~s}, \mathrm{CH}_{3}\right) ; 16.3,16.2\left(2 \mathrm{~d}, 4 \mathrm{CH}_{3},{ }^{3} J_{C P}\right.$ $\left.=6 \mathrm{~Hz},{ }^{3} J_{C P}=6 \mathrm{~Hz}\right)$;

${ }^{31} \mathbf{P}-\mathbf{N M R}\left(\mathbf{C D C l}_{3}, \boldsymbol{\delta}\right): 12.0(\mathrm{~d},=\mathrm{CP}, J=95.59 \mathrm{~Hz})$; $15.0(\mathrm{~d}, \mathrm{CHP}, J=95.59 \mathrm{~Hz})$;

HRMS (ES+) calculated for $\left[\mathrm{M}+\mathrm{Na}^{+}\right]$367.1051, found 367.1053 .

General Procedure for the synthesis of tetraethyl(1-methoxyprop-2-ene-1,2-diyl)bisphosphonate 5

To a solution of tetraethyl (3-bromoprop-1-ene1,2-diyl)bisphosphonate $(\boldsymbol{E})-\mathbf{1} \quad(0.2 \mathrm{~g}, \quad 0.5 \mathrm{mmol})$ diluted in $5 \mathrm{~mL}$ of absolute methanol, was added dropwise tertiary amine $(2 \mathrm{mmol})$ diluted in $3 \mathrm{~mL}$ of methanol at room temperature. The reaction mixture was stirred at reflux for 3 to 6 days (Table 3). After completion, the excess of the solvent was evaporated under reduced pressure and the crude product 5 was purified by column chromatography on silica gel $\left(\mathrm{CH}_{2} \mathrm{Cl}_{2}-\mathrm{MeOH}, 9.5-0.5\right)$.

Yellow liquid;

${ }^{\mathbf{1}} \mathbf{H}-\mathbf{N M R}\left(\mathbf{C D C l}_{\mathbf{3}}, \boldsymbol{\delta}\right): 6.49\left(\mathrm{dd}, 1 \mathrm{H},{ }^{3} \boldsymbol{J}_{H P}=48 \mathrm{~Hz}\right.$, $\left.{ }^{4} J_{H P}=6 \mathrm{~Hz},=\mathrm{CH}\right) ; 6.45\left(\mathrm{dd}, 1 \mathrm{H},{ }^{3} J_{H P}=24 \mathrm{~Hz}\right.$, $\left.{ }^{4} J_{H P}=3 \mathrm{~Hz},=\mathrm{CH}\right) ; 4.32\left(\mathrm{dd}, 1 \mathrm{H},{ }^{2} J_{H P}=15 \mathrm{~Hz},{ }^{3} J_{H P}\right.$ $=9 \mathrm{~Hz}, \mathrm{CHP}) ; 4.23-4.09\left(\mathrm{~m}, 8 \mathrm{H}, 4 \mathrm{OCH}_{2}\right) ; 3.43$ $\left(\mathrm{s}, \mathrm{OCH}_{3}\right) ; 1.35,1.33,1.28(3 \mathrm{t}, 12 \mathrm{H}, J=6 \mathrm{~Hz}, J=6$ $\left.\mathrm{Hz}, J=6 \mathrm{~Hz}, 4 \mathrm{CH}_{3}\right)$;

${ }^{13} \mathbf{C}-N M R\left(\mathbf{C D C l}_{3}, \boldsymbol{\delta}\right): 135.2\left(\mathrm{t},=\mathrm{CH}_{2},{ }^{2} J_{C P}={ }^{3} J_{C P}=\right.$ $7.5 \mathrm{~Hz}) ; 134.3\left(\mathrm{~d},=\mathrm{C},{ }^{1} J_{C P} 177.75 \mathrm{~Hz}\right) ; 74.7(\mathrm{dd}$, CHP, $\left.\quad{ }^{1} J_{C P}=168 \mathrm{~Hz},{ }^{2} J_{C P}=15.75 \mathrm{~Hz}\right) ; 63.2,63.0$ $\left(2 \mathrm{~d}, 2 \mathrm{OCH}_{2},{ }^{2} J_{C P}=7.5 \mathrm{~Hz},{ }^{2} J_{C P}=7.5 \mathrm{~Hz}\right) ; 62.5,62.2$ $\left(2 \mathrm{~d}, 2 \mathrm{OCH}_{2},{ }^{2} J_{C P}=6 \mathrm{~Hz},{ }^{2} J_{C P}=6 \mathrm{~Hz}\right) ; 58.8\left(\mathrm{~d}, \mathrm{OCH}_{3}\right.$, $\left.{ }^{3} J_{C P}=12.75 \mathrm{~Hz}\right) ; 16.4,16.2\left(2 \mathrm{~d}, 4 \mathrm{CH}_{3},{ }^{3} J_{C P}=6 \mathrm{~Hz}\right.$, $\left.{ }^{3} J_{C P}=6 \mathrm{~Hz}\right)$;

${ }^{31} \mathbf{P}$-NMR $\left(\mathbf{C D C l}_{3}, \boldsymbol{\delta}\right): 16.0(\mathrm{~d},=\mathrm{CP}, J=26.62 \mathrm{~Hz})$; $18.2(\mathrm{~d}, \mathrm{CHP}, J=26.62 \mathrm{~Hz})$;

HRMS (ES+) calculated for $\left[\mathrm{M}+\mathrm{H}^{+}\right] 345.12265$, found 345.12183 .

\section{References}

1- $\quad$ L. Aiwen, L. Xiyan, Org. Lett., 2000, 2, $2357-$ 2360.

2- (a) M. Hagithara, N. J. Anthony, T. J. Stout, J. Clardy, S. L. Schreiber, J. Am. Chem. Soc., 1992, 114, 6568-6570; (b) S. Devadder, P. Verheyden, H. C. M. Jaspers, G. Van Binst,D. Tourué, Tetrahedron Lett., 1996, 37, 703-706.

3- Y. Yamamoto, M. Schmid, J. Chem. Soc., Chem. Commun., 1989, 1310-1312.

4- R. Yamaguchi, M. Moriyasu, M. Kawanisi, J. Org. Chem., 1985, 50, 287-288.

5- R. M. Borzilleri, X. Zheng, R. J.Schmidt, J. A. Johnson, S. H. Kim, J. D. Marco, C. R. Fairchild, J. Z. Gougoutas, F. Y. F. Lee, B. H.
Long, G. D. Vite, J. Am. Chem. Soc., 2000, 122, 8890-8897.

6- S. Laschat, H. Kunz, J. Org. Chem., 1991, 56, 5883-588.

7- (a) D. L. Wright, J. P. Schulte II; M. A. Page, Org. Lett., 2000, 2, 1847-1850; (b) F. X.

Felpin, S. Girard, G. Vo-Thanh, R. J. Robins, J. Villiéras, J. Lebreton, J. Org. Chem., 2001, 66, 6305-6312; (c) R. P. Jain, R. M. Williams, J. Org. Chem., 2002, 67, 6361-6365; (d) R. Di Fabio, G. Alvaro, B. Bertani, D. Donati, S. Giacobbe, C. Marchioro, C. Palma, S. M. Lynn, J. Org. Chem., 2002, 67, 7319-7328; (e) P. V. Ramachandran, T. E. Burghardt, L. Bland-Berry, J. Org. Chem., 2005, 70, 7911-7918; (g) P. Besada, L. Mamedova, C. J. Thomas, S. Costanzi, K. A. Jacobson, Org. Biomol. Chem., 2005, 3, 2016-2025.

8- (a) K. C. Nicolaou, M. O. Frederick, R. J. Aversa, Angew. Chem. Int. Ed., 2008, 47, 7182-7225; (b) S. P. Roche, J. A. Porco Jr., Angew. Chem. Int. Ed., 2011, 50, 4068-4093.

9- (a) G. Bartoli, C. Cimarelli, E. Marcantoni, G. Palmieri, M. Petrini, J. Org. Chem. 1994, 59, 5328-5335; (b) M. Liu, M. P. Sibi, Tetrahedron, 2002, 58, 7991-8035; (c) S. M. Hecht, Acc. Chem. Res., 1986, 19, 383-391.

10- (a) Y. -F. Wang, T. Izawa, S. Kobayashi, M. Ohno, J. Am. Chem. Soc., 1982, 104, 6465-6466; (b) S. Hashiguchi, A. Kawada, H. Natsugari, Perkin Trans I, 1991, J. Chem. Soc., 1991, 2435-2444; (c) G. Cardillo, C. Tomasini, Chem. Soc. Rev., 1996, 25, 117-128.

11- (a) E. L. Eliel, X. -C. He, J. Org. Chem., 1990, 55, 2114-2119; (b) Y. Hayashi,; J. J. Rode, E. J. Corey, J. Am. Chem. Soc., 1996, 118, 5502-5503; (c) M. Genov, V. Dimitrov, V. Ivanova, Tetrahedron: Asymmetry, 1997, 8, 3703-3706.

12- (a) E. F. Kleinmann, Comprehensive Organic Synthesis, B. M.,Trost, (Ed.), Pergamon: New York, NY, 1991; pp 893-951; (b) E. J. Corey, G. A. Reichard, Tetrahedron Lett., 1989, 30, 5207-5210.

13- (a) S. Ben Gharbia, R. Besbes,J. Villiéras, H. Amri, Synth. Commun., 1996, 26, 16851692; (b) S. Hbaïeb, Z. Latiri, H. Amri, Synth. Commun., 1999, 6, 981-988; (c) H. Kraiem, I. M. Abdullah, H. Amri, Tetrahedron Lett., 2003, 44, 553-555; (d) A. Arfaoui, F. Béji, T. Ben Ayed, H. Amri, Synth. Commun., 2008, 38, 3717-3725; (e) A. Fray, J. Ben Kraïem, H. Amri, Heteroatom Chem., 2013, 24, 460-465; (f) A. Arfaoui, H. Amri, Synth. Commun., 2015, 45, 2627-2635; (g) K. Jebali, A. Planchat, H. Amri, M. Mathé-Allainmat, J. Lebreton, Synthesis, 2016, 48, 1502-1517. 
14- M. Ameur, A.Arfaoui, H. Amri, Arkivoc, 2014, (v), 199-209.

15- D. Banerjee, R. V. Jagadeesh, K. Junge, H. Junge, M. Beller, ChemSusChem, 2012, 5, 2039-2044.

16- (a) K. Kobayashi, K. A. Miyazama, H. Terrahara, H. Mishime, H. Kurihare, Tetrahedron Lett., 1976, 17, 537-540; (b) R. R. Rsndo,; F. J. Bangerter, Am. Chem. Soc., 1976, 98, 6762-6764; (c) R. R. Rando, F. Bangaeter, J. Am. Chem. Soc., 1977, 99, 5141-5145; (d) R. D. Allan, G. A. R. Johnstone, B. Twitchin, Neurosci. Lett., 1977, 4, 51-54, CA 1977, 86, p 15039o.

17- (a) T. Hashimoto, S. Kondo, H. Naganawa, T. Takita, K. Maede, H. Umezawa, J. Antibiot.,
1974, 27, 86-87; (b) T. Hashimoto, S. Takahashi, H. Naganawa, T. Kakita, H. Umezawa, J. Antibiot., 1972, 25, 350-355; (c) C. Y. P. Teng, B. Garmen, Tetrahedron Lett., 1982, 23, 313-316.

18- T. Kondo, H. Nakai, Tetrahedron, 1973, 29, 1801-1806.

19- D.; E. Natalie, S. C. Donna, M. Khurana, N. S. Noha, P. S. James, J. H. Sylvia, N. Abraham, S. T. Robert, A. M. Jacqueline, Eur. J. Med. Chem., 2003, 38, 49-64.

20- G. Dannhardt, A. Bauer, Nowe, U. J. Prakt. Chem., 1998, 340, 256-263.

21- D. L. Boger, T. Ishizaki, J. R. Wysoki, S. A. Munk, O. Suntornwat, J. Am. Chem. Soc., 1989, 111, 6461-6463. 